\title{
BMJ Open Brazilian network of COVID-19 during pregnancy (REBRACO: a multicentre study protocol)
}

Maria Laura Costa (10 , ${ }^{1}$ Renato T Souza, ${ }^{2}$ Rodolfo C Pacagnella, ${ }^{2}$ Silvana F Bento, ${ }^{3}$ Carolina C Ribeiro-do-Valle, ${ }^{3}$ Adriana G Luz, ${ }^{4}$ Giuliane J Lajos, ${ }^{4}$ Silvia B Mazon, ${ }^{3}$ Luis Bahamondes, ${ }^{3}$ Fernanda G Surita (D) , ${ }^{5}$ Guilherme M Nobrega (i) , 3 Thayna B Griggio, ${ }^{3}$ Charles M'poca Charles (D) , ${ }^{2}$ Maria J Miele, ${ }^{3}$ Ricardo P Tedesco, ${ }^{6}$ Karayna G Fernandes, ${ }^{3,6}$ Sergio Martins-Costa, ${ }^{7}$ Frederico JA Peret, ${ }^{8}$ Francisco E Feitosa, ${ }^{9}$ Rosiane Mattar, ${ }^{10}$ Evelyn Traina, ${ }^{10}$ Edson V Cunha Filho, ${ }^{11}$ Janete Vettorazzi, ${ }^{11}$ Samira M Haddad, ${ }^{3}$ Carla B Andreucci, ${ }^{12}$ José Paulo Guida, ${ }^{13}$ Mario D Correa Junior, ${ }^{14}$ Marcos Augusto Bastos Dias, ${ }^{15}$ Leandro G Oliveira, ${ }^{16}$ Elias F Melo Junior, ${ }^{17}$ Carlos AS Menezes, ${ }^{18}$ Marília GQ Luz, ${ }^{19}$ Jose Guilherme Cecatti (D) , ${ }^{3}$ REBRACO Study Group

To cite: Costa ML, Souza RT, Pacagnella RC, et al. Brazilian network of COVID-19 during pregnancy (REBRAC0: a multicentre study protocol). BMJ Open 2021;11:e051284. doi:10.1136/ bmjopen-2021-051284

- Prepublication history and additional supplemental material for this paper are available online. To view these files, please visit the journal online (http://dx.doi.org/10.1136/ bmjopen-2021-051284).

Received 15 March 2021 Accepted 24 November 2021

Check for updates

(c) Author(s) (or their employer(s)) 2021. Re-use permitted under CC BY-NC. No commercial re-use. See rights and permissions. Published by BMJ.

For numbered affiliations see end of article.

Correspondence to Dr Maria Laura Costa; mlaura@unicamp.br

\section{ABSTRACT}

Introduction The aim of this study was to evaluate the clinical, epidemiological and laboratory aspects of SARSCoV-2 infection during pregnancy and postpartum in 16 maternity hospitals.

Methods and analysis A prospective multicentre study, with five axes. First, the prevalence of SARS-CoV-2 infection among women admitted for childbirth will be described in a cross-sectional study. Second, maternal and perinatal outcomes will be assessed in a prospective cohort study including pregnant or postpartum women with suspected COVID-19. Third, a cohort of positive COVID-19 cases with sampling of a variety of biological material. Histopathological and viral analysis of biological maternal and neonatal samples will be performed, and the assessment of nutritional variables to evaluate the association between vitamin D and severity of infection. Fourth, a monitoring and evaluation committee to collect relevant healthcare information and plan actions in centres facing the pandemic. Furthermore, qualitative studies will be performed to study pregnant women, their families and health professionals. Fifth, an ecological study will monitor the number of live births, stillbirths and other outcomes to explore any trend among the periods before, during and after the pandemic. Data will systematically be collected in an electronic platform following standardised operational procedures. For quantitative study components, an appropriate statistical approach will be used for each analysis. For qualitative data, in-depth interviews recorded in audio will be transcribed, checking the text obtained with the recording. Subsequently, thematic analysis with the aid of the NVivo programme will be performed.

Ethics and dissemination Ethical approval was obtained (letters of approval numbers 4.047.168, 4.179.679 and 4.083.988). All women will be fully informed to sign the consent form before enrolment in the study. Findings will be disseminated through peer-reviewed journals and scientific conferences.

\section{Strengths and limitations of this study}

Consequences of the SARS-CoV-2 infection during pregnancy and postpartum are still not fully understood, especially in low-income and middle-income settings.

- Brazil's data on COVID-19 presented an extremely high number of maternal deaths, especially postpartum, with reported delayed healthcare.

- Early in the pandemic, the Brazilian Network for Studies in Reproductive and Perinatal Health, a team put together over 10 years ago, with experience in multicentre studies in maternal morbidity and preterm birth, established a collaborative multicentre approach in Brazil, Latin America, collaborating with WHO to understand impacts of the COVID-19 in pregnancy.

- The Rede BrAsileira em Estudos do COVID-19 em Obstetrícia study will perform extensive biological sample collection of COVID-19-positive cases, in order to add to current gaps in knowledge considering maternal-fetal transmission and postpartum risks of infection.

- A study protocol proposed during a pandemic of a new infection with uncertain consequences must consider that other relevant interventions might be implemented and available, such as vaccination. The coordinating team will be aware and prompt to consider amendments to the initial protocol and new sets of variables, if necessary.

- The REde BRAsileira em estudos do COVID-19 em Obstetrícia (REBRACO) study will perform extensive biological sample collection of COVID-19-positive cases, in order to add to current gaps in knowledge considering maternal-fetal transmission and postpartum risks of infection. 


\section{INTRODUCTION}

SARS-CoV-2 is a high-impact emerging infectious agent. ${ }^{1}$ The respiratory nature, high rate of transmission and lethality of the virus have made it a global health emergency. ${ }^{2}$ Globalisation and the constant exchange of people in short periods, in addition to multifactorial social, cultural and political-economic aspects, have aggravated the dissemination of this pathogen. This panorama culminated on 11 March 2020, when the WHO classified COVID-19 as a pandemic. ${ }^{2}$ The expansion of this pathogen is factual and its consequences for human healthcare will be felt in a significant way.

Due to the relatively restricted number of COVID-19 reports in the pregnant population, especially among middle-income and low-income (LMI) settings, it is still unclear how the infection caused by this pathogen behaves in its entirety. Some peculiarities of the infection will only be verified through comprehensive longitudinal studies, with a high number of cases monitored. Basic aspects are still controversial, such as the risk of vertical transmission, ${ }^{34}$ differences in disease severity, consequences on different pregnancy trimesters, overall prevalence of infection and a possible increased risk of adverse outcomes. ${ }^{5}$

Studies with a large number of pregnancies affected by COVID-19 in high-income settings have suggested that there is an increased risk of maternal admission to intensive care units (ICUs) and intubation in severe disease. Furthermore, women with previous underlying health conditions and non-white and Hispanic women also have worse outcomes. However, studies did not demonstrate an increased risk of death in comparison to non-pregnant women of reproductive age..$^{5}$ Nevertheless, studies published from Brazil, using national health reports, have presented a different outcome, with a very high number of maternal deaths and evident delays, since most women did not receive any respiratory support and ICU admission for management. ${ }^{6-8}$

Considering perinatal outcomes, early gestational age at infection, low birth weight and maternal ventilatory support are the main determinants of adverse outcomes among cases of COVID-19 infection. ${ }^{9}$

The long-term consequences of the pandemic are still poorly understood, although it is known that there are increased rates of caesarean delivery, delays in the diagnosis of other obstetric complications of relevance, such as hypertension, and possibly increased rates of stillbirth, abortion and prematurity. ${ }^{1011}$ Difficulties in implementing online and remote follow-up of cases, as suggested in many guidelines as an option to avoid hospital exposure, ${ }^{12}$ delays in scheduling imaging and laboratory tests and the fear of seeking medical care in settings known to have an increased number of COVID-19 cases, are of key importance in LMI settings. There is also the impact on healthcare professionals, the way each health facility faces the pandemic, availability of personal protective equipment (PPE), testing tools, and hospital beds for moderate and severe cases.
All those must be addressed to properly understand the impact of the pandemic, particularly in Brazil, a continental size country with huge social and economic disparities.

Early in the pandemic, the Brazilian Network for Studies in Reproductive and Perinatal Health, a team put together over 10 years ago, with experience in multicentre studies in maternal morbidity and preterm birth, ${ }^{13}{ }^{14}$ established a collaborative multicentre approach in Brazil, some Latin American countries and Mozambique. The aim of the REBRACO (in Portuguese: Rede Brasileira em Estudos do COVID-19 em Obstetrícia or Brazilian Network in COVID-19 studies in Obstetrics) initiative is to evaluate the clinical, epidemiological and laboratory aspects related to SARS-CoV-2 infection during pregnancy and the postpartum. In addition, its aim is to make a qualitative assessment of women and healthcare professionals experiencing such a situation, to identify maternal and perinatal outcomes and collect relevant information to provide a quick response and proper organisation of health services to combat the COVID-19 pandemic. In collaboration with WHO and Centro Latinoamericano de Perinatología or Latin American Centre of Perinatology), this initiative will possibly also be expanded to other Latin American countries and Mozambique.

\section{Objectives}

The aim of this study is to address the effects of the COVID-19 pandemic on the obstetric population and related challenges in the fight against the pandemic. The specific objectives were to

1. Describe the prevalence of SARS-CoV-2 infection in a sample of women admitted for childbirth.

2. Characterise clinical, laboratory, maternal and perinatal outcomes, in women with flu-like and/or suspected symptoms of SARS-CoV-2.

3. Analyse the presence of SARS-CoV-2 in different tissues and biological samples.

4. Create preparedness and response plans to tackle the crisis by building a research network at the time of the health emergency.

5. Analyse the indirect effects of the COVID-19 pandemic on reproductive health and the associated factors.

6. Analyse the occurrence of depressive symptoms and post-traumatic stress in pregnant patients with COVID-19, their families and professionals involved in the care of these cases.

7. Identify markers of mortality and prognosis in critically ill patients with COVID-19.

8. Analyse the epidemiological spread of COVID-19 in pregnant women, including geographical dispersion and the influence of social vulnerability indicators.

9. Development of artificial intelligence models for risk prediction, both of viral contamination and clinical complications derived from COVID-19.

10. Analyse the nutritional status, vitamin D serum level and also intestinal microbiome at the time of 


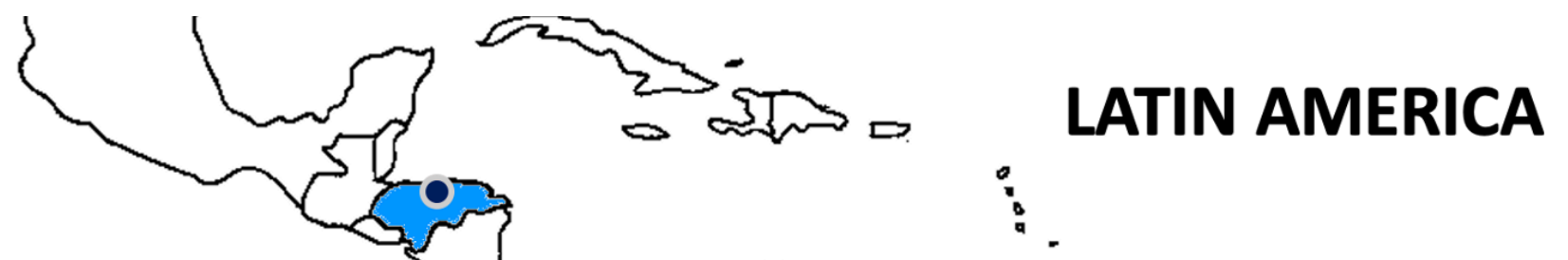

AFRICA

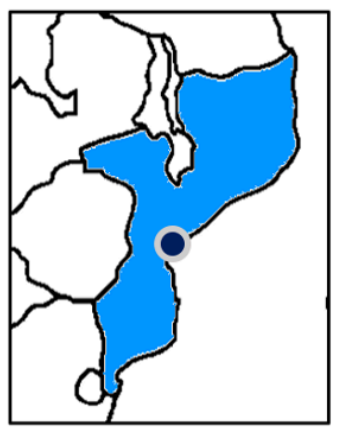

Mozambique
1 REBRACO Centre

2 REBRACO Centres

7 REBRACO Centres to conduct this research protocol (figure 2). Different centres may be included in all or any of the proposed axes, as agreed with the coordinating centre (University of Campinas), depending on the availability of infrastructure, human resources, financial resources and approval by the local research ethics committees at the moment of implementation.

The group has already published a letter, a call for action, to share the initial rationale for this collaboration. ${ }^{15}$
To answer the objectives proposed by this study, the Methods/design section is divided into five axes used 


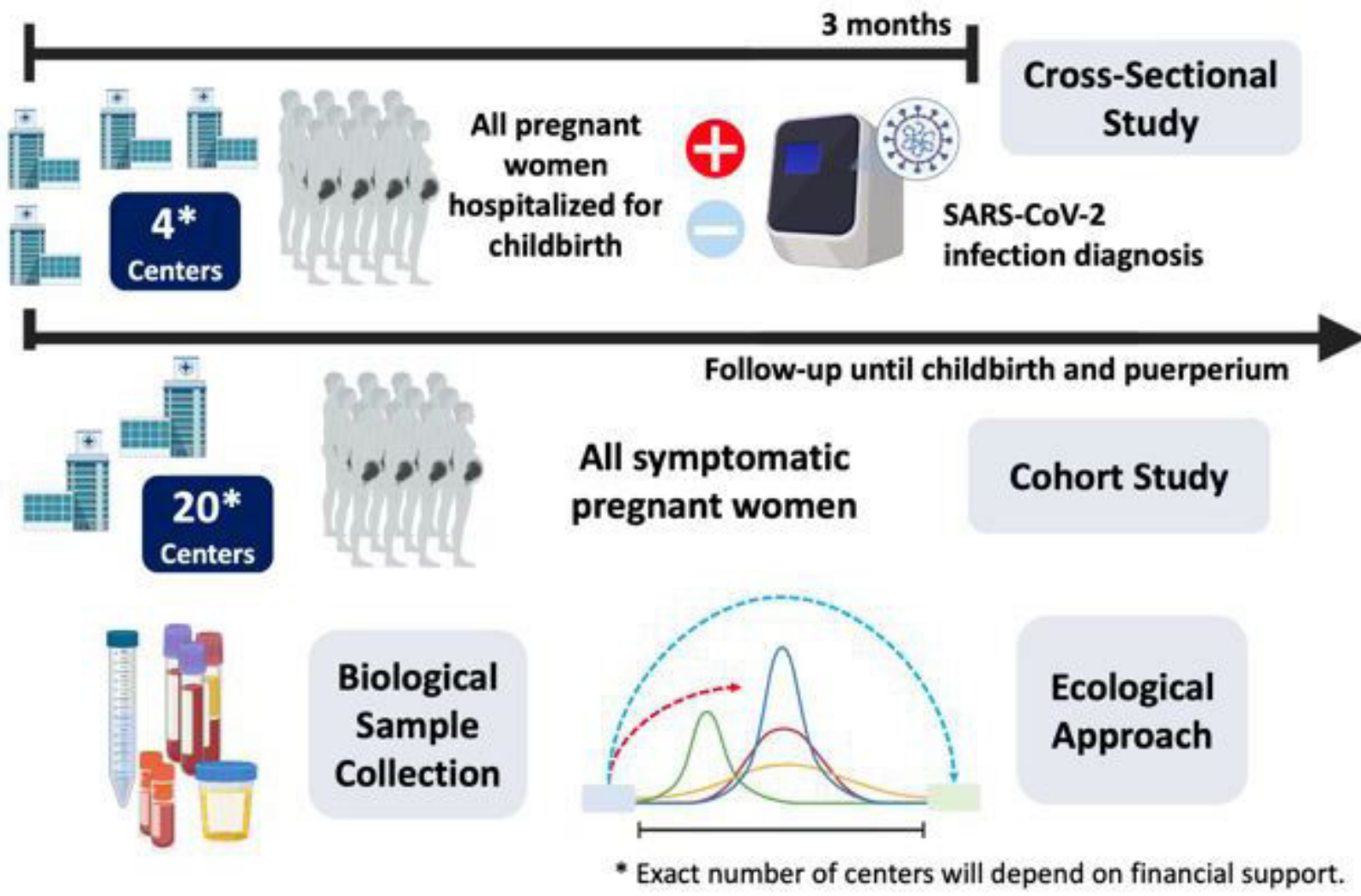

Figure 2

Figure 2 Representative figure of the axes considered for the study

The coordinating centre and each participating centre obtained ethical approval for the study (letter of approval numbers 4.047.168, 4.179.679 and 4.083.988).

\section{Axis 1: cross-sectional study}

A cross-sectional study will be conducted. All parturients from selected centres will be tested for SARS-CoV-2 during 3 months. Clinical admissions will also be tested since women with comorbidities or obstetrical complications that need a hospital admission are potentially at increased risk of delivery. Cases with a positive diagnosis will also have different biological samples collected. These samples will be used to study viral presence and mechanisms of infection associated with adverse outcomes.

In this phase of the study, all pregnant women admitted for childbirth in the participating centres for this component will be included. Initially, the coordinating centre (University of Campinas-CAISM), University Hospital of Jundiaí (HU/FMJ) and Clinics Hospital of Porto Alegre plan to participate. The number of participants was estimated at 1300 (considering an average of 150 deliveries per month in the three maternity hospitals considered, during 3 months). Pregnant women from other collaborating centres may be included with the agreement of the coordinating centre and approval of the local ethics committee. Newborns will also be evaluated. Samples will be collected after the informed consent form (ICF) is accepted and signed. Pregnant women who are not in labour or hospitalised (no indication of delivery) or asymptomatic (without flu-like symptoms) will be excluded from analysis. In Brazil, the national guideline for diagnosis and management of COVID-19 during pregnancy launched on August 28 recommends universal screening but has still not been fully implemented. ${ }^{16}$

All pregnant women included in the cross-sectional study will be tested for SARS-CoV-2. For this, molecular (quantitative reverse transcriptase PCR (RT-qPCR)) detection of SARS-CoV-2 will be performed from respiratory samples of nasopharyngeal/oropharyngeal secretion using swabs or lavage. Every respiratory sample will be stored in duplicate, for diagnostic and research purposes. For the molecular detection, the PCR by realtime RT-qPCR for specific identification of SARS-CoV-2 specific RNA stretches will be performed and results will be made available ideally in less than 24 hours.

All results of viral detection will be shared with the attending physician, and all procedures must be approved by the local healthcare team (and the infection prevention and control teams). The Biorepository for the study will be the Laboratory for the Study of Emerging Viruses (LEVE) at the Institute of Biology, with a BSL-3 
biosafety infrastructure. During this period, all samples must be stored in a biofreezer at $-80^{\circ} \mathrm{C}$ to preserve the biological material in its entirety for reliable molecular detection. Dry ice will be used to transport all samples sent from other centres to preserve the sample during transportation.

Women with positive tests, after/during delivery, will have samples of amniotic fluid, placenta, umbilical cord blood, maternal blood, urine, vaginal secretion, faeces and breast milk collected (for virology research). Likewise, the neonate's combined nasopharyngeal and oropharyngeal samples will be collected (for virology investigation). Sample collection will be detailed in axis 3 of this protocol.

Clinical and sociodemographic information will be obtained by medical record review and included in the RedCap system. Through this platform, protected by a specific password for each researcher, information will be stored, building the database. Each case included will be identified by a number that will be associated with clinical information and the result of the molecular search for infection. Demographic information (age, city of residence, number of previous pregnancies and complications in the current pregnancy) will be obtained, in addition to the presence or absence of the following symptoms in the last 7 days: fever (temperature $>37.8^{\circ} \mathrm{C}$ ), cough, shortness of breath, sputum production, nasal congestion, sore throat, coryza, dysgeusia or dysosmia, and other less typical symptoms, such as abdominal pain and diarrhoea. The selected variables follow the proposed data collection suggested by the WHO, enabling future comparisons and aggregations to other databases and studies. The RedCap database will be further detailed in axis 2 of this protocol.

The home address of these women will also be obtained at the time of entry into the described health facilities. With this information, epidemiological studies of transmission and dissemination of the virus can be performed based on georeferencing and dates, such as the onset of symptoms. These databases will be treated in software such as QGIS, producing georeferenced analyses that will assist in screening for the disease.

\section{Axis 2: cohort study}

Recruitment timing in relation to pregnancy assessment and test/screening results may be implemented differentially, depending on the facility and staffing resources at each study site.

An observational, cohort, prospective study, initially with participant inclusion was planned for at least 6 months, possibly expanding, in case the pandemic progresses. In this study, we will characterise clinical, laboratory, maternal and perinatal outcomes, as well as describe aspects of SARS-CoV-2 infection in pregnancy and in the postpartum of women with flu-like and/or suspected symptoms of SARS-CoV-2, compared with other flu-like syndromes and identify the main severity markers in hospitalised pregnant women admitted to the ICU with SARS from COVID-19, compared with SARS from other causes. The COVID-19 infection will be confirmed based on the laboratory and/ or radiological pulmonary findings, if any positive test for SARS-CoV-2 (either a rapid test or RT-qPCR) or a radiological finding of ground-glass opacities. Women who had a negative test (RT-PCR or rapid test) and did not have ground-glass opacities if submitted to radiological investigation will be considered negative for COVID-19 infection.

The sample size for this study will be stipulated by convenience, composed of all women who meet the inclusion criteria during the study period. Given the lack of solid studies on the prevalence and incidence in the pregnant population at the moment this research protocol was planned and the expected differences due to seasonality and other specificities of transmission, we estimate that around 1000 women will be monitored throughout pregnancy until the puerperium. Again, it all depends on the characteristics of the pandemic and patient demand in the selected health facilities. The participating centres in Brazil will be as follows:

1. CAISM, Centre for Integral Assistance to Woman's Health, University of Campinas, Campinas/SP, Brazil.

2. University Hospital from the Jundiaí Medical School - HU/FMJ, Jundiaí/SP, Brazil.

3. Clinics Hospital from the Federal University of Rio Grande do Sul, Porto Alegre/RS, Brazil.

4. UNIMED Maternity of Belo Horizonte, Belo Horizonte/MG, Brazil.

5. Federal University of Ceará, MEAC/UFC, Fortaleza/ CE, Brazil.

6. São Paulo Hospital, Federal University of São Paulo, UNIFESP/EPM, São Paulo/SP, Brazil.

7. Moinhos de Vento Hospital -HMV, Porto Alegre/RS, Brazil.

8. Jorge Rossmann Regional Hospital, Sócrates Guanaes Institute, Itanhaém/SP, Brazil.

9. Santa Casa de São Carlos, Federal University of São Carlos/UFSCAR, São Carlos/SP, Brazil.

10. Sumaré State Hospital - HES, Sumaré/SP, Brazil.

11. Clinics Hospital, Federal University of Minas Gerais, HC/UFMG, Belo Horizonte/MG, Brazil.

12. Fernandes Figueira Institute, IFF/Fiocruz, Rio de Janeiro/RJ, Brazil.

13. Botucatu School of Medicine, State University of São Paulo, UNESP, Botucatu/SP, Brazil.

14. Clinics Hospital from the Federal University of Pernambuco, HC/UFPE, Recife/PE, Brazil.

15. Climério de Oliveira Maternity, Federal University of Bahia, Salvador/BA, Brazil.

16. Santa Casa de Misericórdia of Pará, Belém/PA, Brazil.

Pregnant women who have criteria for flu-like syndrome (as defined by the Brazilian Ministry of health in the COVID-19 context) during the study data collection period (suspected COVID-19) will be included, after consenting and signing the ICF (and assent form, in cases under 18 years). Cases will be identified in the emergency department, the outpatient clinics or during hospitalisation of women in the included centres. 
Fever and/or at least one respiratory sign or symptom will be considered criteria for the flu-like syndrome: cough, shortness of breath, sputum production, nasal or conjunctival congestion, difficulty swallowing, sore throat, runny nose, $\mathrm{O}_{2}$ saturation $<95 \%$, signs of cyanosis, flapping of the nose and dyspnoea. Other symptoms such as diarrhoea, anosmia and dysgeusia will also be considered. In addition, symptomatic women who participated in axis 1 and asymptomatic women with a positive test will also be included in axis 2 .

Clinical, laboratory, demographic and perinatal results will be retrieved in detail. In addition, information will be obtained regarding women's hospitalisation, the occurrence of severe maternal morbidity and near miss criteria. Molecular research results for the diagnosis of SARSCoV-2 will also be obtained, according to the protocol of each institution. Data will be stored in a decoded form in RedCap, in a database protected by the specific password of the researchers. In patients with a higher severity of disease (SARS), with confirmation or not of COVID-19, we will collect laboratory and clinical information for the use of already known instruments of organ dysfunction and prediction of death in ICUs (SOFA, qSOFA, APACHE, MSI, Maternal Near Miss and CIPHER criteria), in addition to severity markers for COVID-19 already identified in studies in the general population $(\mathrm{C}$ reactive protein, liver enzymes, D-dimer, leucocyte count, platelet count and renal function). Women followed up in the cohort should also be contacted up to 1 year after delivery to assess functionality and psychological status, including post-traumatic stress disorder.

The follow-up of cohort participants will be held with the support of an online application that is currently under development. This tool will be used as a mobile communication strategy to improve maternal and perinatal health. It is based on clinical algorithms of automatic responses and will serve as a strategy to contact patients during cohort follow-up. During the study, the researchers in charge will constantly monitor the tool.

Based on the theoretical prevalence of symptoms from the literature and clinical expertise, a multicriteria decision approach is applied to build a risk score to support patient diagnosis of COVID-19. For the care of patients with respiratory symptoms, the system is parameterised to predict a chance of patients being contaminated with COVID-19 based on their symptoms. The data reported by patients will feed a database that will be used to refine the risk score by means of a supervised machine learning approach. We will use a machine committee composed of decision trees, neural networks and XGBoost algorithms to estimate a chance of each patient having a specific disease or identifying a serious condition. We will then combine the output from the machine learning with the clinical risk score to estimate a poll prevalence of the disease for the set of symptoms.

\section{Axis 3: biological sample collection}

This is another cross-sectional component of the study to obtain samples of women with a confirmed diagnosis of SARS-CoV-2 infection who participated in axes 1 and 2. After these women give their informed consent for sample inclusion in the biorepository study, a nasopharyngeal secretion, blood, urine, vaginal secretion, faeces, placenta, umbilical cord blood, breast milk and newborn nasopharyngeal and oropharyngeal samples will be collected and properly stored in the biorepository. This axis of the project is planned to be implemented in centres according to their ability to collect and store, depending on local infrastructure and funding. The initial expectation is to include the coordinating centre and at least three to four other centres.

Samples will be stored in a biorepository located in the LEVE at the Institute of Biology of UNICAMP, under conditions suitable for sample stability and biosafety. Analyses will be performed, depending on the biosafety level linked to the assay and conducted in biosafety level 2 (BSL-2) environments with due precautions or biosafety level 3 (BSL-3) laboratories, as described later in the biosafety item. The blood centre has an adequate BSL-2 structure and the LEVE, UNICAMP Biology Institute, has both BSL-2 and BSL-3 infrastructures to carry out the analyses. Samples from the other centres will be stored in a local biorepository and afterwards, properly analysed in local laboratories and/or transported to the competent UNICAMP laboratories for analysis. In addition to respiratory samples, the following will be stored:

- Blood: $20 \mathrm{~mL}$ in two EDTA tubes (plasma and leucocytes) and $10 \mathrm{~mL}$ in a dry tube (serum), by peripheral venipuncture.

- Placenta: samples will be collected to guarantee representativeness and random sampling, considering four regions equidistant from umbilical cord insertion, from the central part of the placenta (villous tissue). This sampling procedure will follow the already established protocols, with adaptations and following all biosafety guidelines. ${ }^{17}$ After sampling, the placenta will be sent for histopathological analysis conducted for morphological characterisation and classified according to the Amsterdam consensus. ${ }^{18}$

- Umbilical cord blood: $1 \mathrm{~mL}$ in two tubes of EDTA (plasma and leucocytes), $2-3 \mathrm{~mL}$ in a dry tube (serum), inside the operating room.

- Amniotic fluid: samples of amniotic fluid will be collected preferably at the time of delivery/labour in the operating room, avoiding contamination with maternal blood or vaginal secretion.

- Breast milk: breast milk samples will be obtained from the first milk (colostrum), with prior local hygiene, preferably before breastfeeding begins.

- Urine: $10 \mathrm{~mL}$ of medium jet urine, after intimate hygiene performed by the patient herself, in a sterile universal collection bottle.

- Vaginal secretion: collected using a sterile vaginal swab, preferably during the infection. Samples will not be collected during labour or postpartum.

- Faeces: 25-100g of stool distributed in a sterile universal collection bottle. 
Samples will be collected and processed, at a maximum interval of 4 hours, stored in a biofreezer at $-80^{\circ} \mathrm{C}$. Each sample will be accommodated according to physical conditions, whether it is liquid or solid. These measures are essential to maintain the best conditions of the sample and to conduct further study.

\section{Biosafety}

All procedures of sample collection, processing and diagnosis will follow biosafety protocols considering the intrinsic characteristics of the biological materials included in the project and processing procedures required. ${ }^{1920}$ The entire sample collection and processing team must wear appropriate PPE provided by the study, including disposable surgical mask, disposable apron, disposable gloves, disposable cap, eye protection (glasses or face mask) and closed shoes. When collecting respiratory samples from a patient with suspected or confirmed COVID-19 with the possibility of aerosol spread, the use of an N95 mask is recommended.

Non-propagative diagnostic laboratory work, including sequencing and analysis based on nucleic acids (RTqPCR), in clinical samples from patients with suspected or confirmed SARS-CoV-2 infection, should be performed by adopting appropriate practices and procedures in laboratories (BSL-2). Such facilities are present in CAISM, LPC/HC/UNICAMP, and LEVE-IB/UNICAMP laboratories. Handling of material with high viral concentrations, such as viral propagation, isolation or neutralisation tests, or large volumes of infectious materials, with potential aerosol formation, should only be carried out by appropriately trained and competent personnel in level 3 biosafety laboratories (BSL-3). Such facilities are present in LEVE-IB/UNICAMP.

After handling samples, the work surfaces and equipment used will be decontaminated, with sodium hypochlorite, $62-71^{\circ}$ Gay-Lussac (GL) ethanol, $0.5 \%$ hydrogen peroxide, quaternary ammonium compounds and/or phenolic compounds, according to the manufacturer's recommendations. All residues that come into contact with the samples are destined for infectious waste, and due care associated with this disposal will be taken.

All patient samples from suspected or confirmed SARSCoV-2 cases must be transported, when outside of hospital and laboratory facilities, such as category UN3373, 'Biological Substance Category B'. Viral or isolated cultures must be transported as Category A UN2814, 'infectious substance affecting humans'.

\section{Nucleic acid extraction and quality control}

For samples of respiratory origin and liquid character, SARS-CoV-2 genome extraction will use specific, highprecision commercial kits. For viral RNA extraction, the Quick-RNA Viral (Zymo) or QIAamp Viral RNA Mini (Qiagen) kits will be used, depending on availability, following the recommended protocols. The RNA obtained will be aliquoted for storage in a biofreezer at $-80^{\circ} \mathrm{C}$ and destined for viral detection.
For tissue samples, such as placental, nucleic acid extraction will be performed with the TRIzol Plus RNA Purification kit (Invitrogen). The TRIzol reagent added to the sample, followed by its maceration into crystal zirconia beads, will be used to facilitate RNA extraction. From this, the material will be transferred for RNA extraction by the PureLink RNA Mini Kit (Invitrogen), following the specific protocol for this type of sample. The product of this process will be stored in cryotubes to maintain preservation by cooling or for use in subsequent analysis.

The samples obtained from the biorepository may be processed for the research and characterisation of SARSCoV-2 infection in its laboratory or auxiliary laboratory at the University of Campinas, according to the appropriate technique previously described for viral identification, also allowing for DNA extractions. The integrity and global quantification of any extraction product will be analysed by the NanoDrop equipment (Thermo Fisher).

\section{SARS-CoV-2 RT-qPCR}

For the viral detection of SARS-CoV-2, the RT-qPCR assay will be applied. The technology used for the application is the TaqMan Fast Virus 1-Step Master Mix (Thermo Fisher). A pair of oligonucleotides (forward and reverse primers) designed will be used to characterise the presence of the virus and viral load in the corresponding sample by detecting the fluorescence of TaqMan probes. Owing to the characteristic of the method, the retrotranscriptase phase will occur in the same reaction, due to the nucleic nature of the viral genome (RNA), generating a complementary DNA strand that will be amplified by PCR in real time (qPCR). The use of this type of technology increases the reliability of the result since detection occurs by annealing the probes in a specific amplified sequence, and not as intercalant technology (Sybr), which non-specifically binds to amplified doublestranded DNA. The protocol to be used is based on the following conditions: $30 \mathrm{~s}$ at $48^{\circ} \mathrm{C}$, followed by 45 cycles of denaturation at $95^{\circ} \mathrm{C}$ for $15 \mathrm{~s}$ and annealing-extension at $60^{\circ} \mathrm{C}$ for $1 \mathrm{~min}$. Coupled with the run of the sample in question, a standard curve will be generated from serial dilution of a known concentration of the viral genome to be detected.

\section{Respiratory infection panels and metagenomic analysis}

To assess the presence of other possible viruses in biological samples, in addition to SARS-CoV-2, respiratory infections and metagenome based on new-generation sequencing (NGS) will be analysed. The panels are based on multiplex PCR techniques to qualitatively analyse the presence of several viral and non-viral pathogens. Commercial trials include widely dispersed viral pathogens (such as adenovirus, coronavirus 229E, coronavirus HKU1, coronavirus OC43, coronavirus NL63, human metapneumovirus, human rhinovirus/enterovirus, influenza A, influenza A/H1, influenza A/H1-2009, influenza A/H1-2009, influenza A/H1-2009, influenza A/H1-2009, 
influenza B, parainfluenza 1-4 and Respiratory Syncytial Virus (RSV) and bacterial (Bordetella pertussis, Chlamydophila pneumoniae and Mycoplasma pneumoniae).

For metagenome, NGS refers to non-Sanger-based DNA sequencing technologies, with a high capacity for data formation, which will be used. Millions and even billions of DNA strands can be sequenced in parallel, producing substantially more productivity and minimising the need for the fragment cloning methods that are often used in genome sequencing by Sanger.

Nucleic acids from different samples, analysed one by one, will be sequenced by the platform used and available. This platform will generate ultra-high-throughput data that will be used in metagenomic analyses. Sequences obtained from sequencing will undergo processing after treatment using some bioinformatics programmes and software. The results generated characterise the presence of viral, bacterial and fungal diversity of the samples, enabling analysis of coinfection by other respiratory agents, raising the possibility of severe COVID-19, in association with other viral infections.

\section{Vitamin D evaluation}

The serum level of $25(\mathrm{OH}) \mathrm{D}$ will be determined after 3 hours of fasting. Concentrations will be analysed by highperformance liquid chromatography and UV reading. ${ }^{21}$ Following extraction, the sample, antibody and tracer will be incubated for $90 \mathrm{~min}$ at $20^{\circ} \mathrm{C}-25^{\circ} \mathrm{C}$. Phase separation is accomplished after a $20 \mathrm{~min}$ incubation period at $20^{\circ} \mathrm{C}-$ $25^{\circ} \mathrm{C}$ with the secondary antibody. A buffer is then added before centrifugation to reduce non-specific binding. Radioactivity is measured by a gamma counter and is inversely proportional to the $25(\mathrm{OH}) \mathrm{D}$ concentration in the sample. Liquid chromatography coupled to tandem mass spectrometry, which is considered the gold standard, will be the method used. This method is capable of discriminating between the two forms of vitamin D: one found mainly in vitamin D3 (cholecalciferol) is synthesised mostly by the action of solar ultraviolet radiation on the skin, and the other is vitamin D2 (ergocalciferol), which is of exogenous origin only.

For serum levels, vitamin D 25(OH)D level will be categorised $\mathrm{as}^{22}$ severe deficiency: $<20 \mathrm{nmol} / \mathrm{L}$, deficiency: $<50 \mathrm{nmol} / \mathrm{L}$, insufficiency: $50-150 \mathrm{nmol} / \mathrm{L}$ and desirable: $>100 \mathrm{nmol} / \mathrm{L}$.

\section{Dietary intake}

An adapted Frequency Food Questionnaire (FFQ) will be adapted from the Harvard Willett Food Frequency Questionnaire to trace sources rich in vitamin $\mathrm{D}$ according to the food group: in nature or minimally processed, processed food and ultraprocessed food regarding NOVA Food reference. ${ }^{23}$ For adequate food serving, the FFQ will consider the Brazilian reference for pregnant women. ${ }^{24}$ The Brazilian food table will be chosen to assess and compare micronutrient adequacy. The National Nutrient Database for Standard Reference of the US Department of Agriculture will be used and the industry food label will also be referred. All values will be analysed using the Guideline Dietary Reference Intake for Vitamin D (DRIs). We will categorise as insufficiency, adequate or excessive the levels according to the DRIs: estimate average requirement or adequate intake and tolerable upper intake level. The total energy intake will be calculated and the source of industrial food levels will be assessed. Questionnaires will be online and self-applied by the electronic data storage system RedCap, as previously mentioned.

We will study if there is an association between vitamin D deficiency and/or insufficiency and maternal outcomes according to disease severity. As control variables, we will assess the maternal body mass index, supplementation intake, maternal age and maternal skin colour. In Brazil, race/ethnicity determinants are evaluated through selfreported skin colour because of the miscegenation of the population. Traditionally, the categories of skin colour according to the Brazilian Institute of Geography and Statistics are white, black, pardo, yellow and indigenous.

To compare vitamin levels and disease severity, the outcomes will be classified into four levels of severity ${ }^{25}$ :

1. Mild clinical features without pneumonia diagnosis.

2. Confirmed pneumonia on chest CT with fever and other respiratory symptoms.

3. Hypoxia (oxygen saturation $\leq 93 \%$ ) and respiratory distress or abnormal blood gas analysis results $\left(\mathrm{PaCO}^{2}>50 \mathrm{~mm} \mathrm{Hg}\right.$ or $\left.\mathrm{PaO}^{2}<0 \mathrm{~mm} \mathrm{Hg}\right)$.

4. Respiratory failure requiring intensive care monitoring. Considering the frequency of vitamin D deficiency $(<50 \mathrm{nmol} / \mathrm{L})$ as $16 \%$ in the pregnant population, ${ }^{26}$ assuming a type I error of $5 \%$ as appropriate, considering an $\mathrm{OR}=3$ and type II error of $20 \%$, the total size for this study component should be approximately 70 women per group. A total of 140 participants would be the minimum number required for this analysis. All women included in the cohort study will be invited to consent to receive a WhatsApp call with the link to request the FFQ surveys on RedCap platform.

Descriptive statistics will be calculated and presented as frequencies ( $\mathrm{n}$ and \%), mean and $\mathrm{SD}$, or median with sample characteristics. To compare sources of nutrients according to industrial food processing between intake of two vitamin D levels, adherence to normal distribution using Quantile-quantile plots (Q-Q) plots and histograms followed by a test for normal distribution of numerical variables will be performed. An appropriate statistical analysis will be used for categorical variables, as the $\chi^{2}$ test or the Mann-Whitney or Student t test for numerical variables to compare anthropometrics and maternal differences between vitamin D levels. The Kolmogorov-Smirnov, Shapiro-Wilk and AndersonDarling normality tests will also be used as required, in addition to logistic regression analysis with the estimate of OR and $95 \%$ CI to assess factors related to outcomes (symptom severity). To study the correlation between vitamin D levels and COVID-19 symptoms, the Spearman correlation coefficient will be applied. All results with a $\mathrm{p}$ value below 0.05 will be considered significant. The 


\section{Monitoring and Evaluation Committee}

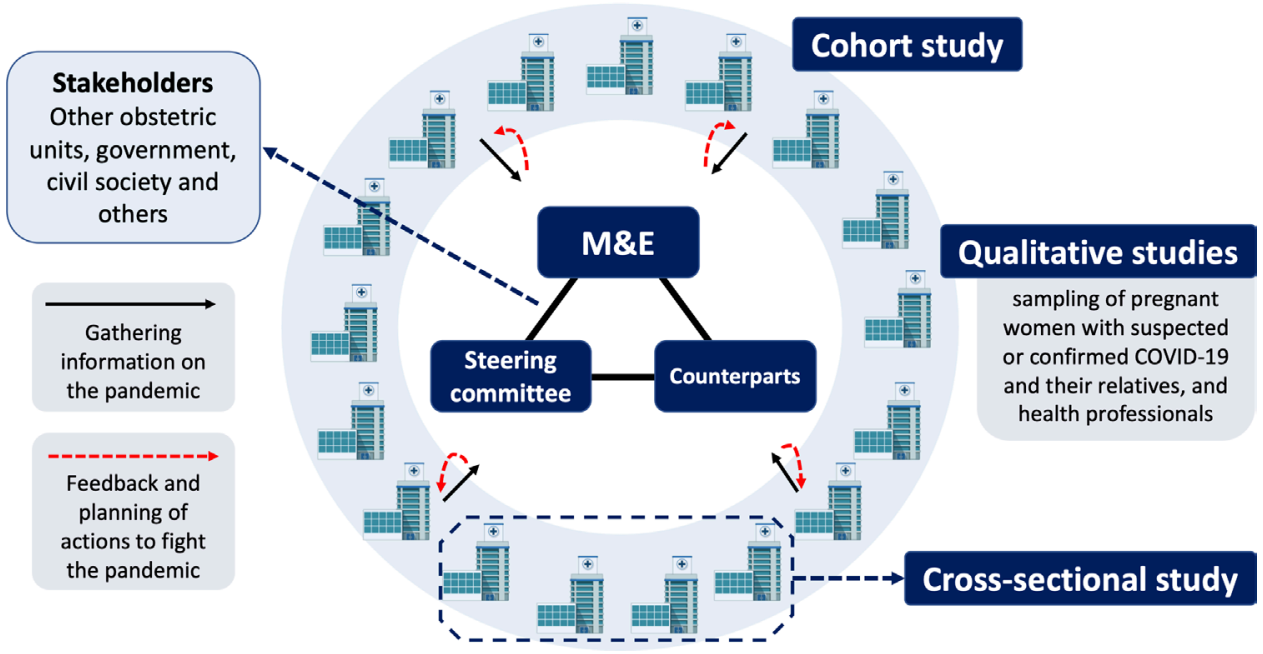

Figure 3 Axis 4-crisis management committee in the COVID-19 Research Network and identification of relevant information to face the pandemic. M\&E, monitoring and evaluation.

software/programme R V.3.6.1 will be used (R Core Team, 2019). ${ }^{27}$

\section{Axis 4: crisis management committee in the COVID-19 research network and identification of relevant information to face the pandemic}

The establishment of the REBRACO initiative (Brazilian network for the study of COVID-19 during pregnancy) goes beyond a clinical-epidemiological study. Its fundamental objective is to assist in tackling the first pandemic wave of the SARS-CoV-2 virus in the Brazilian context and regional context of Latin America by strengthening preparedness and response capacity.

We will implement a monitoring and evaluation (M\&E) management committee to identify information relevant to the following matrices (figure 3): (1) organisation of health services participating in REBRACO; (2) barriers and facilitators during implementation of healthcare and training of healthcare professionals; (3) information and guidance for the general public, pregnant women and their families and healthcare professionals; (4) frequency of flu-like syndrome and SARS related and unrelated to SARS-CoV-2 in pregnant women attended in urgent/ emergency care services, proportion of severe morbidity and maternal mortality, frequency of adverse and related perinatal outcomes and prevalence of flu-like syndrome, severe respiratory syndrome and COVID-19 contamination in parturient women during the pandemic; (5) how pregnant women, their families and health professionals are dealing with the SARS-CoV-2 pandemic, how it influences healthcare and the ability to cope with the pandemic.

An M\&E management plan will be formulated, which will be fundamentally based on periodic actions to monitor real-time data for the five matrices, to make decisions that can help redirect the organisation of health services and healthcare plans, inform the parties involved about ways of coping with the pandemic, identify the need for corrective actions and analyse objectively and systematically the barriers and facilitators, using predetermined process and outcome instruments and indicators. For this purpose, a team of project collaborators will be appointed to compose an M\&E management team that will be responsible for developing, implementing and coordinating the activity plan. The COVID-19 network will encourage the direct or indirect participation of members of counterpart institutions, stakeholders or direct and indirect beneficiaries of the project, benefiting the comanagement of activities and their respective impact and scope results.

Assessment instruments and criteria, monitoring parameters and other M\&E processes will be based on the Monitoring and Evaluation Manual developed by the Pan American Health Organisation. ${ }^{28}$ The M\&E management instruments to be used will be technical meetings, technical reports, performance reports and a final evaluation report.

Weekly technical meetings will be essential for proper implementation, development and monitoring of the project to improve coping skills. The implementation, monitoring and quality committee will be responsible for technical discussions. M\&E committee members may use relevant information to formulate the technical reports, acting as agents for the correction of activities foreseen by the implementation, monitoring and quality committee.

The technical M\&E report will be structured based on the work plan, results, indicators, goals and activities of the project and aims to evaluate the execution and progress of the activities given the results identified in the five matrices in real time. The main sections of this instrument include the description of the results, indicators, goals, actions developed, lessons learnt/recommendations, financial execution and general considerations of 
the M\&E committee, employees and counterparty. The performance report will help assess the project's execution regarding levels of relevance, effectiveness, efficiency, impact and sustainability of the project.

\section{Survey of information relevant to coping with the COVID-19 pandemic}

The development of axes 1, 2, 3 and 5 of this project will inform relevant data to the M\&E committee (which mainly refer to matrix 4): frequency of influenza and SARS-related and unrelated to SARS-CoV-2 in pregnant women seen in urgent/emergency care, the proportion of severe morbidity and maternal mortality, frequency of adverse and related perinatal outcomes, and prevalence of influenza syndrome, SARS and COVID-19 contamination of parturient women during the pandemic.

An approach integrating quantitative and qualitative methods including pregnant women, family members, employees of the COVID-19 network (managers) and healthcare professionals will raise information about the following matrices: (1) organisation of participating health services in the COVID-19 network; (2) barriers and facilitators during implementation of healthcare flows and health professional training; (3) information and guidance for the general public, pregnant women and their families and health professionals; (4) how pregnant women, their families and health professionals are dealing with the SARS-CoV-2 pandemic and how it influences healthcare and coping with the pandemic.

This integrated approach will include quantitative and qualitative studies:

1. Quantitative descriptive study. The intention of this component is to identify strategies implemented in the Obstetric Care service in REBRACO-16 units (participating centres). Managers responsible for the institution participating in this network will be contacted by email and invited to participate. They will complete an online data collection form (Google Form) including information about the institution's structure for obstetric care (number of beds in the emergency room, infirmary and labour wards and ICUs); the number of professionals involved in obstetric care number of doctors, residents, technicians and nurses working in the obstetric care sectors); and qualification of the unit's resources to cope with the pandemic (eg, sufficiency of PPE material, availability of tests). The questionnaire will address this characterisation of participating health services before and during the SARSCoV-2 pandemic.

2. Qualitative study. Three components of qualitative studies will be carried out, focusing on the COVID-19 pandemic experience among pregnant women, their close family members or partners and healthcare professionals. Intentional sampling and saturation sampling will be carried out in all three subgroups of participants, and interviews will be carried out using semistructured interviews properly developed for each particular subgroup. Semistructured interviews will be carried out through open-ended questions, allowing for more in-depth information about the interviewee experience. A single experienced interviewer will conduct all interviews for each subgroup. The interviewers are researchers that are used to conducting qualitative studies. These professionals have some years of experience, with backgrounds as family doctor, psychology and social researcher. A unique list containing three eligible women randomly selected from each participating centre will be used to randomly select those who are to be invited to participate. One additional eligible woman from each centre may be added to the list until reaching the sample size according to the saturation sampling method. An analysis only focused on adolescents (women from 13 to 24 years old) will be carried out. WHO defines adolescence as the population aged 10-19 and the United Nations characterises youth as the period between 15 and 24 years of age. ${ }^{29} 30$ The beginning of adolescence is marked by puberty. Nevertheless, the end of this period is not well defined and continues even after the age of $20{ }^{31}$ This definition of adolescence has an impact on legislation, policies and programmes which are intended to protect and empower adolescents. Therefore, an expanded and more inclusive definition of adolescence as 10-24 years of age would allow society to ensure that investments must be made to support their well-being. ${ }^{31}$ That is why a separate list containing only adolescents and the respective saturation sampling method will be used, following the same methods described earlier. For the health professional's component, we will follow a similar selection process using a list of three randomly selected health professionals from each participating centre, which may include a nursing technician, nurse, assistant professor or medical consultant, and resident in gynaecology and obstetrics.

All semistructured interviews will be recorded on audio after consent from the participants. Subsequently, recordings will be transcribed using Reshape Software, and the text obtained will be checked with the recording. Texts will then be inserted into the NVivo V.12 computer program to help with the analysis. A thematic content analysis will be carried out. ${ }^{32}$

The subgroups and related methods are described further.

\section{Pregnant women}

Pregnant women aged 13-45 years who participated in the cross-sectional study (parturient) or cohort study (in the exposed group) will be eligible to participate. For that purpose, parturient women with positive SARS-Cov-2 tests will be invited to participate in individual telephone interviews during the postpartum period after approximately 15 days since hospital discharge. At that time, they should have already received RT-PCR test results for SARS-CoV2 (PCR subgroups positive and negative for SARS-CoV2). Pregnant women with suspected COVID-19 infection included in the cohort study will be contacted 
to participate by telephone a few days after the initial medical evaluation. In case of hospitalised women, contact will be established after hospital discharge (subgroups of discharged and admitted patients).

Semistructured interviews will address the following topics: information received about the prevention and contagion of SARS-Cov2 and how it applies to your daily life; the impact of a diagnosis or suspected COVID-19 infection in different areas of the woman's life (family life, work and social network); concerns and changes related to self-care; relationship with the partner (when applicable) and members of the family or friends; their experience with the quarantine, including the occurrence of intimate partner violence and economic constraints; what measures could be taken to improve communication and information.

A second telephone interview with women who had a positive COVID-19 test (RT-PCR) from the first subgroup (parturients) will be conducted 60 days after the first contact. Interviews will include the following topics: impact of positive examination on different areas of a woman's life (family life, work and social network); concerns and changes related to self-care and baby care; and the existence of family members with related symptoms or positive SARS-CoV-2 test.

\section{Family member or partner (companion)}

Pregnant women who had a positive COVID-19 test (RTPCR) and participated in the qualitative study will be asked at the end of their interview to indicate a close family member or partner (companion) that could be invited to participate. Only family members or partners aged 18 years or older will be considered. Semi-structured interviews for this component will address the following topics: information received on the prevention and contagion of SARS-CoV-2 and its application to your daily life; the impact of the diagnosis on your family member in different areas of his/her life (family life, work, social network); concerns related to the diagnosis of your relative; what measures could be taken to improve communication and information.

\section{Healthcare professionals}

The qualitative study with healthcare professionals will include nursing technicians, nurses, assistant professors or medical consultants, and residents in gynaecology and obstetrics. Semistructured interviews will address the following topics: information received on the prevention and contagion of SARS-CoV-2 and how it applies to your professional life; information and resources on training and individual protection (source and quality); difficulties encountered during healthcare; concerns related to the COVID-19 pandemic, including the safety of their family; what measures could be taken to provide better communication and information. Data on the sector of activity at the time of the interview, age, time of experience (training), gender, time of work at the unit and schooling will also be collected.

\section{Axis 5: ecological approach}

This is an ecological study on the monthly analysis of health indicators in each participating centre and municipality where the centres are located. We will address the indirect effects of the pandemic on the progression of number of births (number of live births per month), fetal deaths and factors associated with this variation. Other relevant indicators such as preterm birth, caesarean section rates and pre-eclampsia will also be considered. We will collect data before the pandemic, through the pandemic and 12 months after the official end of the pandemic.

Monthly data from each participating centre at each municipality where the REBRACO centres are located will be retrieved from live birth certificates and death certificate notes, and official reports of the surveillance system of each municipality, respectively. All live births and fetal deaths are of compulsory notification and are based on live birth certificates and death certificates of events occurring in the municipality. They are continuously updated in the surveillance system. Death certificates are filled following criteria for fetal deaths standardised according to the Ministry of Health recommendation (fetal losses occurring after 22 weeks or fetal weight $\geq 500 \mathrm{~g}){ }^{33}$ Aggregated sociodemographic, clinical and perinatal data will be also obtained from records of both live birth and death certificates. Data collected via an Excel spreadsheet will be stored in a decoded form in a database, protected under restricted and hierarchical access by a specific password of the researchers.

Furthermore, a trend analysis will be carried out between three periods (12 months before, during and 12 months after the pandemic). The first COVID-19 case was officially announced in Brazil in February 2020.

\section{General procedures}

The participating centres involved in all aspects of the project have adequate conditions to conduct the study. In each participating centre, there will be a researcher responsible for coordinating the study and a research assistant who will receive training before data collection begins. The electronic data storage system (RedCap) is the platform of choice for the study and allows confidentiality and safety in data storage. The RedCap platform server is at the centre coordinating the study (CAISM/ UNICAMP), with a controlled two-way connection between data collection devices (tablets) and the server, which increases safety in data storage. The tablets and installed RedCap application have access restricted by passwords properly created for the study and their handling will be restricted to project activities. There will be partial data extractions, with no interference in running the RedCap data collection platform, aimed at verifying data consistency and informing the M\&E committee and the Implementation, Monitoring and Quality Committee. Reports with inconsistent information will be forwarded to participating centres to double-check data, and correct 
or include any missing information. The committee will carry out the final verification of revised data.

The study will provide different manuals and Standard Operating Procedures (SOP) such as data collection, processing and collection of biological material, filling in the data platform (RedCap database) and conducting the component of qualitative interviews. All research assistants and collaborating researchers will be properly trained through videoconference sessions and webinars facilitated by the implementation, monitoring and quality committee. Weekly meetings and WhatsApp groups between the committee, research assistants and collaborating researchers will help establish communication between the teams and answer questions about training and study procedures.

The study implementation, monitoring and quality committee will have a general monitor and a research assistant to develop the activities provided in the committee's procedures. Activities will be developed directly with the researcher responsible for coordinating the study and his/her research assistants in each collaborating centre.

Considering that throughout the pandemic, other relevant interventions might be implemented and available, such as vaccination, the coordinating team will be aware and prompt to consider amendments to the initial protocol and new sets of variables, if necessary. This is expected when leading with a new and uncertain infection.

\section{ETHICS AND DISSEMINATION}

This research proposal will be guided by all principles set out in the Declaration of Helsinki and Resolution 466/12 of the Brazilian National Health Council. A Biorepository will be instituted within UNICAMP, defined as a collection of human biological material, collected and stored throughout project execution, according to pre-defined technical, ethical and operational regulations or standards, under the management of the principal investigator and institutional responsibility. The biorepository will be installed in the LEVE of the Biology Institute of UNICAMP, coordinated by Profssor Dr José Luiz Proença Modena, with the coresponsibility of the principal investigator of the project, under appropriate regulations. All biorepository institution regulations in force under Resolution 441, of 12 May 2011, ruled by the Brazilian National Health Council, as well as updates, new guidelines, norms and legislation, will be respected. All materials will be under the custody of the principal investigator and will be extended to collaborating researchers and representatives of other centres under express authorisation.

Informed consent for the collection, deposit, storage, use and disposal of human biological material in a biorepository will be formalised using a specific ICF, as recommended in the Brazilian National Health Council resolutions. In case of loss or destruction of samples, as well as closure of the biorepository, the research participant will be notified, and samples will be properly discarded.

To participate in the research, the ICF must be signed. Data will be stored in a database specifically created for this purpose, protected by password in a deidentified manner. For mild cases not admitted to the hospital, due to biosafety reasons, informed consent is possible by telephone contact. The results of the research will be subject to publication in specialised magazines and scientific dissemination congresses. Personal data as well as participant identity will remain confidential.

According to biorepository regulation, also described in the ICF, at the end of the storage period, for up to 10 years, the material of this biorepository may be formally transferred to another biorepository or biobank if authorised by the ethics committee and the participant. Since it is a new disease, considerable doubt surrounds its behaviour during pregnancy. Novel possibilities of further studies may arise over time, and the use of data and biological material collected during the pandemic will be essential to help answer these questions. Any new research that is to be conducted using the stored material will be submitted for approval by the research ethics committee, eventually by the National Research Ethics Committee, should it be applicable.

Other institutions participating in the project, in addition to UNICAMP, will have (depending on funding) structure for the installation and management of a biobank and/or biorepository for biological samples included in the project, after approval by the local ethics committee. All ethical aspects permeating the use of biological samples stored in a biobank and/or biorepository will follow current biosafety rules and legislation, as explained in Resolution 441 of 12 May 2011, regulated by the National Health Council. Sample collection will only be carried out after the participant signs the ICF, as previously stated. In the event of dissolution of partnership between any institutions of the specific proposal associated with the biorepository, samples may be transferred to the UNICAMP Biorepository or may have another destination, depending on the disconnected institution and project coordination, following all pertinent legal aspects. Human biological material can be stored in a biorepository throughout the validity of the current research.

The management component of the management and evaluation committee in the COVID-19 Research Network is part of an implementation component since data will not be collected from managers, except for the eventual data collection on the resources of each institution to combat the pandemic.

In the qualitative approach, pregnant women and parturients who are already included in the quantitative axis of this study will be invited. Initial contact inviting to participate in the survey will be made using the telephone number obtained at the time women registered in this study. Semistructured interviews with pregnant women, parturients and their families will be conducted by telephone and recorded after consent. Telephone interviews 
with health professionals will occur after recorded informed consent. Interviews should last around $20 \mathrm{~min}$.

For the telephone interview, an ICF prepared specifically for this purpose will be read. After clarifying doubts, the participant will be asked if she agrees to participate in the survey and allows these questions to be recorded. If the answer is affirmative, it will be explained that the recorder will be turned on and both questions will be repeated and recorded. Soon after, the interview will begin. The participant will receive a digital copy of the ICF by email.

All participants will be informed that they can refuse to participate in the study or interrupt their participation at any time without any consequences. In underage $(<18$ years old) participants, an authorisation from parents/ legal guardians will be requested for the interview, as well as the adolescent's assent. Participants will be assured that their identity will be kept confidential and no information will be given to others who are not part of the research team. When publicising the results of the study, the names of the participants will not appear. To guarantee confidentiality, cases will be identified by numbers, as well as interview recordings and transcripts.

Ethical approval for the coordinating centre was obtained from IRB (letters of approval numbers 4.047.168, 4.179.679 and 4.083.988; online supplemental material) and then each participating centre also received its local approval. All women will be fully informed to sign an ICF before enrolment in the study. Results will be published in peer-reviewed journals and presented in scientific conferences. Key findings will also be disseminated via various media, including Twitter for the study (@rebraco2020).

\section{DISCUSSION}

Researchers in the Department of Obstetrics and Gynaecology at the University of Campinas have extensive experience in multicentre studies, both at international and national levels. The study will be conducted by the Brazilian Network of Studies on Reproductive and Perinatal Health. It will also have the support of the Centre for Studies on Reproductive Health of Campinas (Cemicamp), a private non-profit organisation that is the research arm of the Department of Obstetrics and Gynaecology of the University of Campinas, with experience in planning, execution and administration of clinical and epidemiological studies and currently selected as a WHO special collaborating centre, within a partnerships through the HRP Alliance (Development and Research Training in Human Reproduction).

Establishing such an initiative in Brazil is not an easy task. It involves experience and capacity in clinical and epidemiological studies, in addition to knowledge and use of 'Good Clinical Practice' standards. This is the reason we chose to include participating centres from different parts of Brazil in the different axes of the study. These centres also have previous experience in the inclusion of women in clinical and epidemiological studies and a previous good record of performance. In one of the recent multicentre studies coordinated by UNICAMP, we published the processes and experiences involved in planning and conducting a multicentre study involving the collection of clinical-epidemiological data and biological samples. ${ }^{34}$ We also explored the barriers and methods adopted to overcome these obstacles. This documented and shared experience exemplifies UNICAMP's role in coordinating studies of this scale. One of the important characteristics described in this article is the fundamental role of institutional commitment to the good development of studies. All participating centres were previously contacted about the possibility of conducting the study, and a commitment was made to assure adequate implementation of the project in all centres.

The pandemic has created considerable challenges for people's lives, including even tighter budgetary constraints, barriers to healthcare access, fears related to the risk of vertical transmission or transmission/infection, concerns about taking care of a baby while sick with COVID-19, mental health disorders, intimate partner and domestic violence, and workers' rights and safety, among others. A rapid response to such a complex and challenging situation is fraught with difficulties. National and international and multicollateral collaborations are essential to address this matter.

Currently, it is not completely known whether pregnant women are more likely to have adverse outcomes resulting from the infection. Maternal and perinatal health can be impacted by infection, but so far, little is known about the impact of SARS-CoV-2 infection on short-term or longterm maternal and perinatal health. Likewise, the effects of infection on conceptual products such as the placenta and breast milk are largely unknown. Additionally, it is not known whether there is SARS-CoV-2 transmission to the newborn through breast milk, although there is evidence that other viruses are transmitted through breast milk. The current scenario is serious and the psychological repercussions of the pandemic on the population of pregnant women, their families and health professionals involved in the care of patients affected by this pandemic are not fully known. Nevertheless, severe repercussions are presumed to exist.

The importance of a network study that broadens knowledge about SARS-CoV-2 infection during pregnancy is reiterated throughout the panorama described earlier, especially in Latin America, currently one of the major locations where the pandemic is still rampant. Knowledge of the repercussions of this virus is scarce in the obstetric population and needs to be more widespread, both to face the current COVID-19 pandemic and the future repercussions on maternal and perinatal health. The current study will allow an international alliance to standardise the study of COVID-19 in pregnancy and to establish a collaboration to share expertise among health services from different settings of LMIC, building capacity and strengthening their ability to combat the pandemic and implement local policies. The acquisition of real-time 
data and consolidation of information from one or more centres, with a consequent production of relevant scientific knowledge, are crucial to identify barriers and facilitators of the implementation process of local policies dedicated to maternal and childbirth care. Furthermore, information is essential to address the unmet needs related to the unknown rate of disease progression in pregnant women and the short-term repercussions on the fetus/newborn.

\section{Author affiliations}

${ }^{1}$ School of Medical Sciences. Department of Obstetrics and Gynaecology, State University of Campinas Faculty of Medical Sciences, Campinas, Brazil

${ }^{2}$ Department of Obstetrics and Gynaecology, State University of Campinas Faculty of Medical Sciences, Campinas, Brazil

${ }^{3}$ Department of Obstetrics and Gynecology, State University of Campinas Faculty of Medical Sciences, Campinas, Brazil

${ }^{4}$ Obstetrics and Gineacology, State University of Campinas Faculty of Medical Sciences, Campinas, Brazil

${ }^{5}$ Obstetrics and Gyneacology, State University of Campinas Faculty of Medical Sciences, Campinas, Brazil

${ }^{6}$ Department of Obstetrics and Gynecology, Faculdade de Medicina de Jundiai, Jundiai, Brazil

${ }^{7}$ Department of Obstetrics and Gynecology, Universidade Federal do Rio Grande do Sul, Porto Alegre, Brazil

${ }^{8}$ Department of Obstetrics and Gynecology, Unimed Belo Horizonte, Belo Horizonte, Brazil

${ }^{9}$ Department of Obstetrics and Gynecology, Sociedade de Assistencia a Maternidade Escola Assis Chateaubriand, Fortaleza, Brazil

${ }^{10}$ Department of Obstetrics and Gynecology, UNIFESP, Sao Paulo, Brazil

${ }^{11}$ Department of Obstetrics and Gynecology, Hospital Moinhos de Vento, Porto Alegre, Brazil

${ }^{12}$ Department of Obstetrics and Gynecology, UFSCar, Sao Carlos, Brazil

${ }^{13}$ Department of Obstetrics and Gynecology, State University of Campinas, Campinas, Brazil

${ }^{14}$ Department of Obstetrics and Gynecology, UFMG, Belo Horizonte, Brazil

${ }^{15}$ Department of Obstetrics, Instituto Fernandes Figueira, Rio de Janeiro, Brazil

${ }^{16}$ Department of Obstetrics and Gynecology, UNESP Campus de Botucatu, Botucatu, Brazil

${ }^{17}$ University of Pernambuco, Recife, Brazil

${ }^{18}$ Obstetrics and Gyneacology, Salvador University, Salvador, BA, Brazil

${ }^{19}$ Santa Casa de Misericórdia do Pará, Belem, Pará, Brazil

Correction notice This article has been corrected since it was first published. Figure 2 has been updated.

Twitter Maria Laura Costa @laura_unicamp and Maria J Miele @MieleMaju

Acknowledgements Figures were created with BioRender.com.

Collaborators Rede Brasileira em Estudos do COVID-19 em Obstetrícia (REBRACO) Study Group: Sherly Metelus, Lester Castro, Stephanie Pabon, Rachel Soeiro, Amanda D Silva, Paulo S R Junior, Thais G Sardinha, Rodolfo R Japenga, Erica R F Urquiza, Maíra R Machado, Marcela Maria Simões, Larissa M Solda, Jussara Mayrink, Sue Yazaki-Sun, Priscilla Mota, Arimaza C Soares, Ellen Machado, Anne Bergmann, Gustavo Raupp dos Santos, Patrícia B Peres, Cristiane L Arbeli, Rafael M Quevedo, Carolina F Yamashita, Julia D Corradin, Isabella Bergamini, José Geraldo L Ramos, Maria Lúcia R Oppermann, Laisa S Quadro, Lina Marins, Érika V Paniz, Thaís Vicentini Xavier, Bruna E Parreira, Aline Tosetto, Sabrina Savazoni, Aline C Costa, Marina HL Almeida, Bruna FV Moura, Lidiane R França, Hanna Vieira, Rafael B Aquino, Débora F Leite, Isabella Monteiro, Marcos Nakamura-Pereira, Bruna 0 Guerra, Gabriela Gorga, Daisy Pinheiro, Denise Cordeiro, Priscila L Miná, Carol Dornellas, Kevin FA Oliveira, Caio Leal, Rayra Amana, Cristiane 0 Santos, Marina M dos Santos, Carlos Neto, and Thiago Gomes, Isabela R Pereira, Clélia Andrade Salustrino, Valéria B Pontes, Roberto Allen da Silva Franco, João Paolo Bilibio, Gislânia P F Brito, Hana Paula C Pinto, Danielle Leal de Oliveira, Andrezza A Guerra, Andrea 0 Moura, Natasha Pantoja, Fernanda David, Alina Silva and Juliana Vasconcellos Freitas-Jesus. Also, we would like to acknowledge the staff from the coordinator centre which has had a major contribution as part of the REBRACO initiative: Angela M Bacha, Anderson Borovac-Pinheiro, Belmiro G Pereira, Eliana M
Amaral, Elton Ferreira, Helaine MBPM Milanez, Jamil P S Caldas, Luiz F Baccaro, Marcelo Nomura, Patrícia M Rehder, Renata Z Simone, Renato Passini Jr, Cristiano Torrezan, João L P Modena, Magnun N Nunes dos Santos, Sergio T M Marba and Tábata R Zumpano dos Santos.

Contributors The idea for conceptualising the study arose from a discussion among MLC, RCP, RTS and JGC. They initially got the contribution of other members of the coordinating centre including the staff, SBF, CCR-d-V, AGL, GJL, SBM, LB and FGS, and some postgraduate students GMN, TBG, CMC, MJM and JPG. Then the idea of increasing the initiative to a network for a multicentre study arose and RPT, KGF, SM-C, FJAP, FEF, RM, ET, EVCF, JV, SMH, CBA, MDCJ, MABD, LGO, EFMJ, CASM and MGQL were invited and contributed with information for building the proposal. All of them read and agreed on the final form of the manuscript.

Funding Up to now, the study was supported by Fundo de Apoio ao Ensino, à Pesquisa e à Extensão-Unicamp (grant number 2300/20) and also from the Washington University at Saint Louis, USA, McDonnell Academy seed grant for research on infectious diseases and the impact of COVID-19 (grant 'COVID-19 in the Placenta: Understanding the Consequences in Pregnancy', available at https://global.wustl.edu/ mcdonnell-academy-awards-seed-grants-infectious-diseasesimpact-of-covid-19/).

Map disclaimer The inclusion of any map (including the depiction of any boundaries therein), or of any geographical or locational reference, does not imply the expression of any opinion whatsoever on the part of BMJ concerning the legal status of any country, territory, jurisdiction or area or of its authorities. Any such expression remains solely that of the relevant source and is not endorsed by BMJ. Maps are provided without any warranty of any kind, either express or implied.

Competing interests None declared.

Patient consent for publication Consent obtained directly from patient(s) Provenance and peer review Not commissioned; externally peer reviewed. Supplemental material This content has been supplied by the author(s). It has not been vetted by BMJ Publishing Group Limited (BMJ) and may not have been peer-reviewed. Any opinions or recommendations discussed are solely those of the author(s) and are not endorsed by BMJ. BMJ disclaims all liability and responsibility arising from any reliance placed on the content. Where the content includes any translated material, BMJ does not warrant the accuracy and reliability of the translations (including but not limited to local regulations, clinical guidelines, terminology, drug names and drug dosages), and is not responsible for any error and/or omissions arising from translation and adaptation or otherwise.

Open access This is an open access article distributed in accordance with the Creative Commons Attribution Non Commercial (CC BY-NC 4.0) license, which permits others to distribute, remix, adapt, build upon this work non-commercially, and license their derivative works on different terms, provided the original work is properly cited, appropriate credit is given, any changes made indicated, and the use is non-commercial. See: http://creativecommons.org/licenses/by-nc/4.0/.

\section{ORCID iDs}

Maria Laura Costa http://orcid.org/0000-0001-8280-3234

Fernanda G Surita http://orcid.org/0000-0003-4335-0337

Guilherme M Nobrega http://orcid.org/0000-0001-9406-4256

Charles M'poca Charles http://orcid.org/0000-0001-7133-0574

Jose Guilherme Cecatti http://orcid.org/0000-0003-1285-8445

\section{REFERENCES}

1 Andersen KG, Rambaut A, Lipkin WI, et al. The proximal origin of SARS-CoV-2. Nat Med 2020;26:450-2.

2 Organization WH. Coronavirus Disease 2019 (COVID-19) - situation report 128, 2020.

3 Vivanti AJ, Vauloup-Fellous C, Prevot S, et al. Transplacental transmission of SARS-CoV-2 infection. Nat Commun 2020;11:3572.

4 Groß R, Conzelmann C, Müller JA, et al. Detection of SARS-CoV-2 in human breastmilk. Lancet 2020;395:1757-8.

5 Mullins E, Evans D, Viner RM, et al. Coronavirus in pregnancy and delivery: rapid review. Ultrasound Obstet Gynecol 2020;55:586-92.

6 Takemoto M, Menezes MO, Andreucci CB, et al. Clinical characteristics and risk factors for mortality in obstetric patients with severe COVID-19 in Brazil: a surveillance database analysis. BJOG 2020;127:1618-26.

7 Takemoto MLS, Menezes MO, Andreucci CB, et al. Maternal mortality and COVID-19. J Matern Fetal Neonatal Med 2020;14:1-7. 
8 Takemoto MLS, Menezes MdeO, Andreucci CB, et al. The tragedy of COVID-19 in Brazil: 124 maternal deaths and counting. Int $J$ Gynaecol Obstet 2020;151:154-6.

9 Di Mascio D, Sen C, Saccone G, et al. Risk factors associated with adverse fetal outcomes in pregnancies affected by coronavirus disease 2019 (COVID-19): a secondary analysis of the WAPM study on COVID-19. J Perinat Med 2020;48:950-8.

10 Jeong SY, Sung SI, Sung JH, et al. Mers-Cov infection in a pregnant woman in Korea. J Korean Med Sci 2017;32:1717-20.

11 Alserehi H, Wali G, Alshukairi A, et al. Impact of middle East respiratory syndrome coronavirus (MERS-CoV) on pregnancy and perinatal outcome. BMC Infect Dis 2016;16:105.

12 Salsi G, Seidenari A, Diglio J. Obstetrics and gynecology emergency services during COVID-19 pandemic. Am J Obstet Gynecol MFM 2020;100214.

13 Lajos GJ, Tedesco RP, Passini R. Methodological issues on planning and running the Brazilian multicenter study on preterm birth. ScientificWorldJournal 2015;2015:719104-10.

14 Cecatti JG, Costa ML, Haddad SM, et al. Network for surveillance of severe maternal morbidity: a powerful national collaboration generating data on maternal health outcomes and care. BJOG 2016;123:946-53.

15 Costa ML, Pacagnella RC, Guida JP, et al. Call to action for a South American network to fight COVID-19 in pregnancy. Int J Gynaecol Obstet 2020;150:260-1.

16 MINISTÉRIO DA SAÚDE. Manual de Recomendações para a Assistência Gestante E Puérpera frente Pandemia de COVID-19. Brazil: MINISTÉRIO DA SAÚDE, 2020.

17 Nobrega GM, Guida JP, Japecanga RR. Placental sampling for understanding viral infections - a simplified protocol for the COVID-19 pandemic. Rev Bras Ginecol Obstet 2020.

18 Khong TY, Mooney EE, Ariel I, et al. Sampling and definitions of placental lesions: Amsterdam placental workshop group consensus statement. Arch Pathol Lab Med 2016;140:698-713.

19 World Health Organization. Laboratory biosafety guidance related to coronavirus disease 2019 (COVID-19), 2020. Available: https://apps. who.int/iris/bitstream/handle/10665/331138/WHO-WPE-GIH-2020. 1-eng.pdf

20 Organização Pan-americana de Saúde, World Health Organization. Diretrizes provisórias de biossegurança laboratorial para o manuseio e transporte de amostras associadas ao novo coronavírus 2019 (COVID-19) - interim guidance 12 February 2020. Available: https:// iris.paho.org/handle/10665.2/51914

21 Eisman JA, Shepard RM, DeLuca HF. Determination of 25-hydroxyvitamin D2 and 25-hydroxyvitamin D3 in human plasma using high-pressure liquid chromatography. Anal Biochem 1977;80:298-305.

22 Maeda SS, Saraiva GL, Kunii IS, et al. Factors affecting vitamin D status in different populations in the city of São Paulo, Brazil: the São Paulo vitamin D evaluation study (SPADES). BMC Endocr Disord 2013;13:14.

23 Monteiro CA, Levy RB, Claro RM, et al. A new classification of foods based on the extent and purpose of their processing. Cad Saude Publica 2010;26:2039-49.

24 Barbieri P, Nishimura RY, Crivellenti LC, et al. Relative validation of a quantitative FFQ for use in Brazilian pregnant women. Public Health Nutr 2013;16:1419-26.

25 Wang Y, Liu Y, Liu L, et al. Clinical outcomes in 55 patients with severe acute respiratory syndrome coronavirus 2 who were asymptomatic at hospital admission in Shenzhen, China. J Infect Dis 2020;221:1770-4.

26 Figueiredo ACC, Cocate PG, Adegboye ARA, et al. Changes in plasma concentrations of 25-hydroxyvitamin $D$ and 1,25-dihydroxyvitamin D during pregnancy: a Brazilian cohort. Eur J Nutr 2018;57:1059-72.

27 R Core Team. R: a language and environment for statistical computing, 2019. Available: https://www.r-project.org/

28 Pan American Health Organization - PAHO/WHO Brazil Country Office. Manual for the Monitoringmonitoring and Evaluationevaluation of Cooperationcooperation Agreementsagreements / Panpan American Healthhealth Organizationorganization. Brasilia: Pan American Health Organization, 2017. https://www.paho.org/bra/images/stories/GCC/ ingles_manual\%20de\%20monitaramento\%202016.pdf?ua=1

29 World Health Organisation (WHO). Health for the World's Adolescents : A second chance in the second decade, 2014.

30 UN Secretariat/UNESCO/ILO. Definition of youth. world programme of action for youth. Available: http://undesadspd.org/Youth.aspx

31 Sawyer SM, Azzopardi PS, Wickremarathne D. The age of adolescence. Lancet Child Adolesc Heal 2018;4642:1-6.

32 Park N. Qualitative evaluation and research methods. 2nd ed. Newbury Park,CA: Sage, 1990: 1-532.

33 Ministério da Saúde. A declaração de óbito: documento necessário eE importante. Ministério dadA Saúde, Conselho Federalfederal de Medicinamedicina, Centro Brasileiro de Classificação de Doenças. 3rd edn. Brasília: Ministério da Saúde, 2009.

34 Souza RT, Cecatti JG, Costa ML, et al. Planning, implementing, and running a multicentre preterm birth study with Biobank resources in Brazil: the preterm samba study. Biomed Res Int 2019;2019:1-8. 